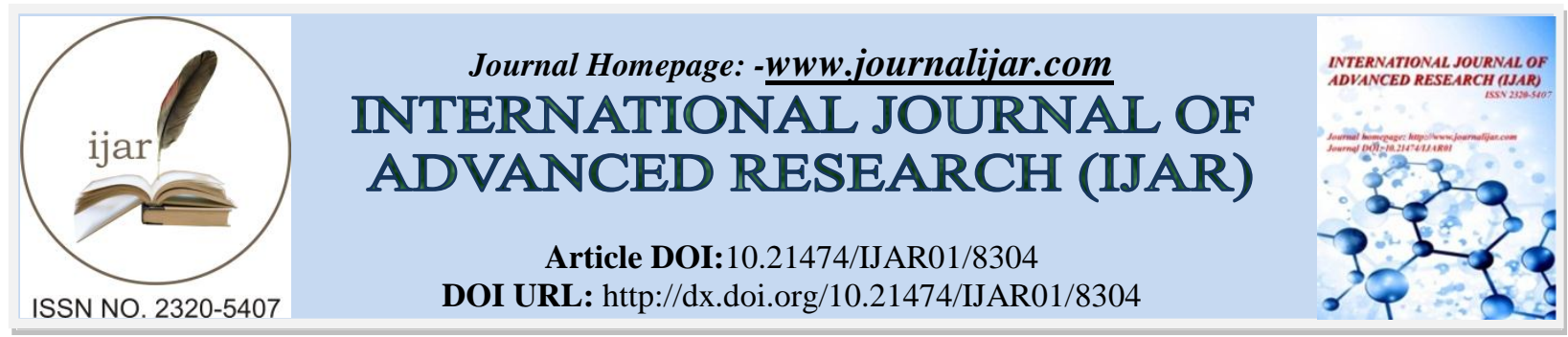

RESEARCH ARTICLE

\title{
RECENT ADVANCES IN PULP CAPPING MATERIALS.
}

Jeslee Ann Jose ${ }^{1}$, Prathap M.S ${ }^{2}$ and K. Harish S. Shetty ${ }^{3}$.

1. Post Graduate Student, Department of Conservative Dentistry And Endodontics, Yenepoya Dental College, Yenepoya Deemed to be University,Mangalore, Karnataka.

2. Professor, Department of Conservative Dentistry And Endodontics, Yenepoya Dental College,Yenepoya Deemed to be University,Mangalore, Karnataka.

3. Professor and Head of the Department, Department of Conservative Dentistry And Endodontics, Yenepoya Dental College, Yenepoya Deemed to be University,Mangalore, Karnataka.

\section{Manuscript Info}

\section{Manuscript History}

Received: 01 November 2018

Final Accepted: 03 December 2018

Published: January 2019

Key words:-

Hydroxyapatite,Bioceramics

,Endosequence,EnamelMatrix

Proteins,Stem cells.

\section{Abstract}

Managing immature teeth with pulp exposures due to extensive caries has become more common, particularly with the introduction of new restorative materials. These new materials have demonstrated the capacity to maintain tissue viability for the duration of dental organ development and closure of the apex of root. Historically, calcium hydroxide has been the medicament of choice for traumatic injuries, according to several publications, as it provided the adequate environment for the formation of mineralized tissue due to its high $\mathrm{pH}$ and disinfecting properties. Currently, with the development of MTA and other bioceramic materials, techniques have evolved and new opportunities exist for treatment instead of extraction. This article aims at reviewing the various newer materials for pulp capping.

Copy Right, IJAR, 2017,. All rights reserved.

\section{Introduction:-}

Endodontic therapy, also known as root canal treatment, is one of the most commonly used techniques in dental clinics. Endodontic therapy is a procedure for removing contaminated or injured dental tissue, refilling, and sealing off the created void with synthetic material to eliminate future contamination. With advancements in antibiotic therapies, dental materials, and endodontic technology, the success rate of endodontic therapy has increased dramatically over the past decade ${ }^{1}$. Current endodontic procedures replace the vital pulp with synthetic materials, rather than living tissue. Extruded endodontic materials can cause a foreign body reaction. Pulpless teeth loose their ability to sense environmental changes, making the progression of caries unnoticeable by patients. Another advantage of maintained dental pulp vitality is to maintain the capacity for limited dentin regeneration ${ }^{2}$.

\section{Objective Of Pulp Therapy:-}

1. To maintain the integrity and health of the teeth and their supporting tissues.

2. To maintain the vitality of the pulp of a tooth affected by caries, traumatic injury, or other causes.

\section{Corresponding Author:-Jeslee Ann Jose.}

Address:- Post Graduate Student, Department of Conservative Dentistry And Endodontics, Yenepoya Dental College, Yenepoya Deemed to be University,Mangalore, Karnataka. 
The results of study done by R. Kemal Su'bay showed that hydroxyapatite does not induce hard tissue bridging at the exposure site in human dental pulp. Moderate infiltration of the inflammatory cells was seen generally in the teeth capped with hydroxyapatite. According to R. M. Frank a continuous, well-calcified bridge was observed and no inflammatory cells were present in the residual pulp. In some cases, denticles containing some synthetic hydroxyapatite crystals were found in the radicular pulp. The calcified bridge was made up by osteodentine and near the pulp, tubular dentine with a layer of predentine and odontoblasts were noted. Odontoblastic processes were located in deninal tubules and the intertubular dentine showed interwoven calcified collagen fibrils. ${ }^{3}$

\section{Bioceramics:-}

Recently, EndoSequence Root Repair Material (ERRM, Brassler, Savannah, GA, USA), BioAggregate (Verio Dental Co, Vancouver, Canada), Biodentin (Septodont, Saint-Maur-des-Fosses, France) and many other bioceramicbased products have been introduced which can be used with the same applications as MTA. Cytotoxicity of ERRM was similar to that of MTA .Biodentin and MTA had also a similar efficacy in pulp-capping treatment. However, further studies are necessary to evaluate these materials in vital pulp therapy. ${ }^{4}$

\section{Endo Sequence Root Repair Material:-}

It consists of:-

1. Calcium silicates

2. Monobasic calcium phosphate

3. Zirconium oxide

4. Tantalum oxide

5. Proprietary fillers

6. Thickening agents

Hirschman et al., compared Cytotoxicity of MTA-Angelus, BrasselerEndosequence Root Repair Putty (ERRP), Dycal and Ultra-blend Plus (UBP)-(light curable $\mathrm{Ca}(\mathrm{OH}) 2)$ and concluded that ERRP and UBP are less cytotoxic. ${ }^{5}$

\section{Biodentin:-}

Biodentin was developed by Septodont's Research Group as a new class of dentin material which could conciliate high mechanical properties, excellent biocompatibility and bioactive behavior. It was developed as a silicate based restorative material. Due to its good sealing ability with dentin, it is used as a dentin replacement material. Biodentin has its use both in coronal part of tooth and in roots ${ }^{6}$. It also has shorter setting time thus eliminating the drawbacks of MTA.It has dentin-like mechanical properties, which may be considered a suitable material for clinical indications of dentin-pulp complex regeneration such as direct pulp capping. It has a positive effect on vital pulp cells and stimulates tertiary dentin formation. ${ }^{7}$

The powder mainly contains tricalcium and dicalcium silicate (3CaO $\mathrm{SiO} 2$ and $2 \mathrm{CaO} \mathrm{SiO} 2$ ), the principal component of Portland cement, as well as calcium carbonate $(\mathrm{CaCO} 3)$. Zirconium dioxide $(\mathrm{ZrO} 2)$ serves as contrast medium. The liquid consists of calcium chloride $(\mathrm{CaCl} 2 . \mathrm{H} 2 \mathrm{O})$, which is used as a setting accelerator and waterreducing agent in aqueous solution with an admixture of polycarboxylate (a superplasticizing agent) According to AlicjaNowickaBiodentine had a similar efficacy in the clinical setting and may be considered an interesting alternative to MTA in pulp-capping treatment during vital pulp therapy ${ }^{8}$.

\section{Setting Mechanism of Biodentin:-}

The calcium silicate has the ability to interact with water leading to the setting and hardening of the cement. During setting there is hydration of the tricalcium silicate $(3 \mathrm{CaO} . \mathrm{SiO} 2=\mathrm{C} 3 \mathrm{~S})$ which produces a hydrated calcium silicate gel (CSH gel) and calcium hydroxide $\mathrm{Ca}(\mathrm{OH}) 2$ ).

$$
\begin{aligned}
& 2(3 \mathrm{CaO} \cdot \mathrm{SiO} 2)+6 \mathrm{H} 20---> \\
& \mathrm{C}_{3} \mathrm{~S} \\
& 3 \mathrm{CaO} \cdot \mathrm{SiO} 2.3 \mathrm{H} 20+3 \mathrm{Ca}(\mathrm{OH})_{2}
\end{aligned}
$$

This dissolution process occurs at the surface of each grain of calcium silicate. The hydrated calcium silicate gel and the excess of calcium hydroxide tend to precipitate at the surface of the particles and in the pores of the powder, due to saturation of the medium ${ }^{9}$. This precipitation process is reinforced in systems with low water content. The unreacted tricalcium silicate grains are surrounded by layers of calcium silicate hydrated gel, which are relatively impermeable to water, thereby slowing down the effects of further reactions. The $\mathrm{C}-\mathrm{SH}$ gel formation is due to the 
permanent hydration of the tricalcium silicate, which gradually fills in the spaces between the tricalcium silicate grains. The hardening process results from of the formation of crystals that are deposited in a supersaturated solution ${ }^{10}$.

\section{Calcium enriched mixture:-}

Calcium enriched mixture (CEM) cement (YektazistDandan, Tehran, Iran) was introduced to dentistry as an endodontic filling biomaterial. ${ }^{11}$

\section{Components:-}

1. Calcium oxide $(\mathrm{CaO})$

2. Sulfur trioxide $\left(\mathrm{SO}_{3}\right)$

3. Phosphorous pentoxide $\left(\mathrm{P}_{2} \mathrm{O}_{5}\right)$,

4. Silicon dioxide $\left(\mathrm{SiO}_{2}\right)$.

The physical properties of this biomaterial, such as flow, film thickness, and primary setting time are favorable, and its clinical applications are similar to those of MTA.

Several animal studies have shown that in various forms of VPT treatments, the induction of dentin bridge formation in teeth treated with CEM was comparable to that of MTA and superior to CH. Studies of complete pulpotomy treatment using CEM, MTA, and $\mathrm{CH}$ have shown that compared to $\mathrm{CH}$, samples in the CEM group exhibited lower inflammation, improved quality/thickness of calcified bridge, superior pulp vitality status, and morphology of odontoblast cells. However, no significant differences were identified in comparison to MTA. ${ }^{12}$

DPC outcomes of prospective randomized clinical trials carried out on 32 permanent premolar teeth that were orthodontically planned for extraction have shown that under immunohistochemical examinations, thickness of dentinal bridge and pulp inflammation beneath CEM was comparable to MTA at various time intervals.

\section{Enzymes:-}

1. Heme-Oxygenase-1:Heme oxygenase-1 (HO-1) activity is related to stem cell differentiation. According to Sun-Ju Kim HO-1 induction by cobaltic protoporphyrin IX (CoPP) in human dental pulp cells (HDPCs) increased cell growth and mineralization. Pharmacologic HO-1 induction might represent a potent therapeutic approach for pulp capping and the regeneration of human dental pulp cells.

2. Simvastatin:According to Kyung-San Min Simvastatin enhanced the differentiation of human dental pulp cells by up-regulating mineralization nodules and odontogenic markers as well as angiogenic markers. ${ }^{13}$

\section{Novel endodontic cement (NEC):-}

Recently, a new endodontic cement (NEC) consisting of different calcium compounds was developed by Asgary. Clinical uses of this cement are similar to MTA. It has good handling characteristics. NEC is also able to produce hydroxyapatite. The results of an in vivo study showed that as pulp capping materials, MTA and NEC showed similar favorable results. These results were better than calcium hydroxide.

\section{NEC consists of:-}

1. Calcium oxide,

2. Calcium phosphate,

3. Calcium carbonate,

4. Calcium silicate,

5. Calcium sulfate,

6. Calcium chloride.

According to SaeedAsgaryno inflammation was observed in MTA and NEC groups, and in $75 \%$ of each, dentinal bridge was completely formed. ${ }^{14}$

\section{Growth factors:-}

Growth factors are peptide molecules that transmit signals between cells functioning as stimulators and/or inhibitors of growth as well as modulators of differentiation state amongst other roles. The potential functional activities of the growth factors sequestrated within dentin matrix may be quite extensive. In addition to the induction of 
odontoblast- like cell differentiation, these molecules may signal cell proliferation and migration within the pulp. A number of reports of the in vivo or in vitro placement of exogenous growth factors, particularly TGF (Transforming Growth Factor) and Bone Morphogenetic Proteins (BMPs), on exposed pulps have demonstrated the potential of these molecules to signal reparative dentinogenic events. Transdentinal or direct application of TGF-1 and BMP-7 to the odontoblasts of unexposed pulps in cultured tooth slices has also shown the ability of these growth factors to signal reactionary dentinogenesis. Pulps capped with BMP-7 demonstrated a hard tissue that was bone-like in appearance. ${ }^{15}$

\section{Bone Sialoprotein (Bsp):-}

Bone sialoprotein (BSP) is a major non-collagenous protein in mineralizing connective tissues such as dentin, cementum and calcified cartilage tissues. BSP is involved in regulating hydroxyapatite crystal formation in bones and teeth. BSP stimulates the differentiation of cells which secrete an organized extracellular matrix more efficiently than any other capping material used so far. Altogether, the results reported here support that bone sialoprotein displays novel bioactive properties and is capable of stimulating in 1 months' time the development of a thick reparative dentinal tissue in the pulp, occluding the perforation and filling the mesial third of the pulp chamber. ${ }^{16}$

\section{Enamel Martrix Derivative:-}

It is known that the enamel matrix proteins play biological roles in the formation of dentine, acellularcementum and alveolar bone during tooth development. Bone sialoprotein and osteopontin are major noncollagenous protein expressed in bone and dentine. Enamel matrix proteins have been reported to increase the levels of mineralization markers (including bone sialoprotein and osteopontin) in odontoblasts. Based on this concept, the enamel matrix derivative (EMD) was formulated.The regenerative process of EMD consists of differentiation of odontoblasts with consequent dentine formation and pulpal wound healing without affecting the vitality of the remaining pulp in a manner similar to normal dentinogenesis .Amelogenin is the principal component of EMD that has an important role in dentine formation during dentinogenesis.

When a pulp wound is exposed to EMD, a significant amount of reparative dentine-like tissue is formed with successive neogenesis of normal pulp tissues, in a manner similar to the classical woundhealing process. This process appears to imitate normal dentinogenesis due to the high fractions of amelogenin and amelin found in EMD. EMD has also been reported to contain growth factors such as transforming growth factor-beta 1 and small amelogenin peptides that are actively involved in cell signalling to stimulate matrix formation and mineralization. These growth factors are recognized as mediators in processes such as tissue homeostasis, inflammation, healing and neogenesis.

\section{Lyophilized Freeze-Dried Platelet-Derived Preparation:-}

Kalaskar and Damle149 compared the efficacy of a lyophilized freeze-dried platelet-derived preparation to that of calcium hydroxide as pulpotomy agents in primary molars. Fifty-six primary molars in 28 children were treated by a conventional pulpotomy technique. Twenty-eight teeth were treated with the lyophilized freeze-dried plateletderived preparation and another 28 with calcium hydroxide. Clinical evaluations were performed at 1,3 and 6 months, and radiographic evaluations were performed at 1 and 6 months. The authors reported that the success rate with the lyophilized freeze-dried platelet-derived preparation was better than that with calcium hydroxide. ${ }^{18}$

\section{Nanohydroxyapatite:-}

Nanohydroxyapatite was used as a pulpotomy and direct pulp capping agent in primary pig teeth by Shayegan et al.161 The results of the their histological study revealed that, in the short-term and in caries-free pig teeth, nanohydroxyapatite appeared to be biocompatible and provoked no moderate or severe inflammatory reaction in pulp tissues with either pulpotomy or direct pulp capping treatments. ${ }^{19}$

\section{Propolis:-}

Propolis is collected from trees and shrubs by honeybees. The main chemical classes present in propolis are flavonoids, phenolics and other various aromatic compounds. Flavanoids are well-known plant compounds which have antioxidant, antibacterial, antifungal, antiviral and anti-inflammatory properties.

Propolis is composed of 50\% resin and vegetable balsam, 30\% wax, 10\% essential and aromatic oils, 5\% pollen and $5 \%$ other various substances, including organic debris. Ansorge has shown the ability of Propolis to stimulate the production of transforming growth factor (TGF) Beta 1 which is important for the differentiation of odontoblasts. It also induces the synthesis of collagen by dental pulp cells. As a natural product, Propolis has demonstrated potent 
antimicrobial and anti-inflammatory properties. Propolis has shown to inhibit synthesis of prostaglandins and supports the immune system by promoting phagocytic activities, stimulating cellular immunity and augmenting healing effects. Additionally, it contains some elements (i.e. zinc and iron) that are important for the collagen synthesis. $^{20}$

\begin{abstract}
Allium Sativum Oil:-
Recently, Mohammad et al compared the clinical and radiographic effects of A. sativum oil and those of formocresol in vital pulpotomies of primary teeth. Their results showed that A. sativum oil had good healing potential, leaving the remaining pulp tissue functioning and healthy. Vital pulpotomy with A. sativum oil had a $90 \%$ success rate, while that with formocresol was $85 \%$. The authors concluded that A. sativum oil is a biocompatible material that is compatible with vital human pulp tissue. It has good healing potential and leaves the remaining pulp tissue healthy and functioning. ${ }^{21}$
\end{abstract}

\title{
Platelet Rich Plasma And Platelet Rich Fibrin:-
}

The conventional method of revitalization procedure was done by inducing bleeding into the pulp canal by mechanically irritating the periapical tissues. In necrotic teeth with open apices, some amount of pulp tissue along with Hertwigs Epithelial Root Sheath may survive apically and these tissues can proliferate once the inflammatory condition are reversed and the canal becomes totally disinfected. The created blood clot acts as a matrix for the in growth of new tissues into the pulp canal. ${ }^{22}$ However, this procedure will cause discomfort for the patient while mechanically irritating the periapical tissues. In the past two decades, an increased understanding of the physiological roles of platelets in wound healing and after tissue injury has led to the idea of using platelets as therapeutic tools. Platelet-Rich Plasma (PRP) consists of a limited volume of plasma enriched with platelets, which is obtained from the patient. $^{23}$

PRF consists of an autologous leukocyte-platelet-rich fibrin matrix composed of a tetra molecular structure, with cytokines, platelets and stem cells within it which acts as a biodegradable scaffold that favors the development of microvascularization and is able to guide epithelial cell migration to its surface ${ }^{24}$. This autologous matrix demonstrated in in vitro studies a great potential to increase cell attachment and a stimulation to proliferate and differentiate osteoblasts. PRF releases cytokines such as IL-4 by activating a subpopulation of T-cells which aids in healing by moderating inflammation and inhibiting IL-1b-mediated stimulation of MMP-1, MMP-3, and synthesis of prostaglandin E-2 thereby inducing regeneration of pulpal tissue. ${ }^{25}$

\section{Stem Cells:-}

Regenerative medicine using stem cells is a promising tool in a new clinical platform for a whole spectrum of intractable diseases. Various stem cells have been reported, including embryonic stem cells (ES cells), induced pluripotent stem cells (iPS cells), and somatic stem cells ${ }^{26}$. Because of ethical problems, immunorejection, and tumorigenesis, ES cells and iPS cells have many issues to overcome. Among these, somatic stem cells, especially mesenchymal stem cells (MSCs) isolated from various tissues including bone marrow, adipose tissue, skin, umbilical cord, and placenta, have been used in clinical applications. have already used bone marrow-derived MSCs (BMMSCs) instead of autogenous bone grafting in clinical trials for the effective treatment of osseous defects, and favourable results have been obtained.

However, bone marrow aspiration is an invasive and painful procedure for the donor. In addition, the number, proliferation, and differentiation potential of BMMSCs decline with increasing age. Dental pulp appears to be an alternative and more readily available source of stem cells. Stem cells from the pulp of permanent teeth (dental pulp stem cells [DPSCs]) and from exfoliated deciduous teeth (SHED) have been identified as a novel population of stem cells that have the capacity of self-renewal and multilineage differentiation ${ }^{27}$.

A previous report showed that DPSCs are able to differentiate into odontoblastic and osteoblastic lineage and generate dentin/pulp complex and bone. Moreover, DPSCs have been reported to have the potential for use in cellbased therapy for systemic disease, such as neurologic disease and cardiac disease, and to ameliorate ischemic disease. The main advantage of using SHED is that it can be obtained noninvasively from deciduous teeth that are routinely extracted in childhood and generally discarded as medical waste without any ethical concerns. Despite extensive reports on DPSCs, the characteristics and gene expression profile of SHED remain poorly understood. ${ }^{28}$ 


\section{Conclusion:-}

Managing immature teeth with pulp exposures due to extensive caries has become more common, particularly with the introduction of new restorative materials. These new materials have demonstrated the capacity to maintain tissue viability for the duration of dental organ development and closure of the apical root. In summary, vital pulp therapy provides an additional viable option for the treatment of immature permanent teeth with vital pulps allowing the preservation of tissue necessary for the maturation and apexogenesis of the tooth. It also allows the possibility to keep teeth that otherwise will be extracted and cause an adverse effect to young growing individuals.

\section{Reference:-}

1. Robertson A, Andreasen FM, Andreasen JO, Noren JG. Long-term prognosis of crownfractured permanent incisors: the effect of stage of root development and associated luxation injury. (2000):Int J Paediatr Dent;10:191-9.

2. Rabie G, Trope M, Tronstad L. Strengthening of immature teeth during long-termendodontic therapy. (1986) Endod Dent Traumatol;2:43-7.

3. Katebzadeh N, Dalton BC, Trope M. Strengthening immature teeth during and afterapexification. (1998):J Endod;24:256 -9.

4. Cvek M. Prognosis of luxatednonvital maxillary incisors treated with calcium hydroxide and filled with gutta percha: a retrospective clinical study. (1992): Endod DentTraumatol;8:45-55.

5. Love RM. Effects of dental trauma on the pulp. (1997):Pract Periodontics Aesthet Dent;9:427-36, 38 (quiz).

6. Shabahang S, Torabinejad M. Treatment of teeth with open apices using mineraltrioxide aggregate. (2000):Pract Periodontics Aesthet Dent;12:315-20, 22 .

7. Webber RT. Apexogenesis versus apexification. (1984):DentClin North Am;28:669 -97.

8. Massler M. Preventive endodontics: vital pulp therapy. (1967):DentClin North Am;Nov:663-73.

9. Kakehashi S, Stanley HR, Fitzgerald RJ. The effects of surgical exposures of dental pulps in germ-free and conventional laboratory rats. (1965):OralSurg Oral Med Oral Pathol;20:340 -9.

10. Kakehashi S, Stanley HR, Fitzgerald RJ. The effects of surgical exposures of dental pulps in germ-free and conventional laboratory rats. (1966):J South Calif Dent Assoc;34:449 -51.

11. Horsted-Bindslev P, Vilkinis V, Sidlauskas A. Direct capping (2003):Oral Surg Oral Med OralPathol Oral Radiol Endod;96:591- 600.

12. Trope M, McDougal R, Levin L, May KN Jr, Swift EJ Jr. Capping the inflamed pulp under different clinical conditions. (2002):JEsthetRestor Dent; 14:349 -57.

13. Kiba H, Hayakawa T, Nakanuma K, Yamazaki M, Yamamoto H. Pulpal reactions to two experimental bonding systems for pulp capping procedures. (2000):J Oral Sci;42:69 -74.

14. Ulmansky M, Sela J, Langer M, Yaari A. Response of pulpotomy wounds in normalhuman teeth to successively applied Ledermix and Calxyl. Arch Oral Biol(1971);16:1393- 8.

15. Schroder U, Granath LE. Scanning electron microscopy of hard tissue barrier followingexperimental pulpotomy of intact human teeth and capping with calcium hydroxide.Odontol Revy (1972);23:211-20.

16. Schroder U. Evaluation of healing following experimental pulpotomy of intact humanteeth and capping with calcium hydroxide. Odontol Revy (1972);23:329-40.

17. Fuks AB. Pulp therapy for the primary dentition. In: Pinkham JR, Casamassimo PS, Fields HW Jr, McTigue DJ,

18. Nowak A, eds. Pediatric Dentistry: Infancy Through Adolescence.(2005) 4th ed. St. Louis, Mo: Elsevier SaundersCo;:375-93.

19. American Association of Endodontists. Glossary of Endodontic Terms. 7th ed. Chicago, Ill: American Association ofEndodontists; (2003).

20. American Association of Endodontists. Systematic en-dodontic diagnosis. Insert to the Fall/Winter edition of Endodontics: Colleagues for Excellence; (1996).

21. McDonald RE, Avery DR, Dean JA. Management of trauma to the teeth and supporting tissues. In: Dentistry for the Child and Adolescent.(2004) 8th ed. St Louis, Mo: Mosby Inc;:455-502.

22. Coll JA, Sadrian R. Predicting pulpectomy success and its relationship to exfoliation and succedaneous dentition.(1996 )Pediatr Dent;18(1):57-63.

23. Camp JH, Fuks AB. Pediatricendodontics: Endodontic treatment for the primary and young permanent dentition.(2006) In: Cohen S, Hargreaves KM, eds. Pathways of the Pulp. 9th ed. St. Louis, Mo: Mosby Elsevier;:834-59.

24. Farooq NS, Coll JA, Kuwabara A, Shelton P. Success rates of formocresolpulpotomy (2000):Pediatr Dent;22(4):278-86. 
25. Fuks AB. Current concepts in vital pulp therapy. (2002):Eur J Pediatr Dent;3(3):115-20.

26. Vij R, Coll JA, Shelton P, Farooq NS. Caries control and other variables associated with success of primary molar vital pulp therapy. (2004):Pediatr Dent;26(3):214-20.

27. Murray PE, About I, Franquin JC, Remusat M, Smith AJ. Restorative pulpal and repair responses. (2001):J Am Dent Assoc;132(4):482-91.

28. Holan G, Eidelman E, Fuks AB. Long-term evaluation of pulpotomy in primary molars using mineral trioxide aggregate or formocresol. (2005):Pediatr Dent;27(2):129-36. 